\title{
Research Article \\ Reinforced Ultra-Tightly Coupled GPS/INS System for Challenging Environment
}

\author{
Xueyun Wang, Kui Li, Pengyu Gao, and Wei Wang \\ School of Instrument Science and Optoelectronics Engineering, Beihang University, Beijing 100191, China \\ Correspondence should be addressed to Kui Li; xflikui@126.com
}

Received 30 June 2014; Revised 24 September 2014; Accepted 24 September 2014; Published 16 October 2014

Academic Editor: Jian Guo Zhou

Copyright (c) 2014 Xueyun Wang et al. This is an open access article distributed under the Creative Commons Attribution License, which permits unrestricted use, distribution, and reproduction in any medium, provided the original work is properly cited.

\begin{abstract}
Among all integration levels currently available for Global Positioning System (GPS) and Inertial Navigation System (INS) Integrated System, ultra-tightly coupled (UTC) GPS/INS system is the best choice for accurate and reliable navigation. Nevertheless the performance of UTC GPS/INS system degrades in challenging environments, such as jamming, changing noise of GPS signals, and high dynamic maneuvers. When low-end Inertial Measurement Units (IMUs) based on MEMS sensors are employed, the performance degradation will be more severe. To solve this problem, a reinforced UTC GPS/INS system is proposed. Two techniques are adopted to deal with jamming and high dynamics. Firstly, adaptive integration Kalman filter (IKF) based on fuzzy logics is developed to reinforce the antijamming ability. The parameters of membership functions (MFs) are adjusted and optimized through self-developed neutral network. Secondly, a Doppler frequency error estimator based on Kalman filter is designed to improve the navigation performance under high dynamics. A complete simulation platform is established to evaluate the reinforced system. Results demonstrate that the proposed system architecture significantly improves navigation performance in challenging environments and it is a more advanced solution to accurate and reliable navigation than traditional UTC GPS/INS system.
\end{abstract}

\section{Introduction}

GPS/INS integrated system is the best choice for many navigation applications and basis composition for most comprehensive navigation systems. New techniques and innovative integration algorithms have been being proposed all the time [1-3]. GPS/INS integrated system can be classified into three types by the integration level, namely, loosely coupled, tightly coupled, and ultra-tightly coupled (also called deeply coupled). Researchers disagree on what exactly defines each of the three couplings [4-6]. The definitions used in this paper are referred to those given by Gao and Lachapelle in [5]. If only GPS tracking is assisted by INS, the GPS/INS system is defined as UTC system. In UTC systems, pseudorange (PR)/pseudorange-rate (PRR) or inphase (I)/quadrature $(\mathrm{Q})$ could be used as measurements in IKF and they are not related to the integration level. Due to the better navigation performance resulted from the advanced data fusion method, UTC GPS/INS system has become a prior solution to accurate and reliable navigation applications in challenging environments. In traditional UTC
GPS/INS systems the mutual assistance between GPS and INS is strengthened so that the signal tracking ability is much improved, but the IKF remains the same. This causes problems when GPS signal is jammed, interfered so that the noise characteristics are dramatically changed. In such cases, the measurement mathematic models in IKF must be adapted to achieve better navigation performance [7]. In another scenario of challenging environments where the vehicle is performing high dynamic maneuvers, the large errors of INS aiding information caused by low-end inertial sensors could lead to degraded navigation performance or even divergence of IKF, because an error positive feedback is formed inside the integrated system after introducing INS aiding [8]. Therefore the adaptation ability and high dynamic property are two important issues for MEMS-based UTC GPS/INS system.

Some investigations have been conducted in related areas. Research was conducted on performance enhancement for ultra-tight GPS/INS system using a fuzzy adaptive strong tracking unscented Kalman filter, verifying the effectiveness of fuzzy logic adaptive control (FLAC) [9]. However the membership functions (MFs) of the FLAC are determined 
empirically without any adjustments and optimizing. Authors examined three adaptive filtering techniques: artificially scaling of the predicted Kalman filter covariance, the adaptive Kalman filter, and multiple model adaptive estimation in [10], and their advantages and disadvantages are detailed and compared. All algorithms were tested with the GPS and inertial data simulation software and results showed that adaptive GPS/INS integrated system could significantly improve the speed of the dynamic alignment and potentially improve the overall navigation accuracy and integrity. The use of fuzzy logic control techniques for Autonomous Underwater Vehicle (AUV) is highlighted in [11]. The adaptation of the initial statistical assumption of KF, which might be inaccurate due to changes in sensor noise characteristics, was expected. The proposed algorithms were implemented to real experimental data from Hammerhead AUV and results showed a promising result in improving the estimation of $\mathrm{KF}$ and enhancing the overall accuracy of the integrated INS/GPS system. Similar conclusions about the advantages of FLAC were also drawn in $[12,13]$. Sun proposed a generalized neutral network to implement fuzzy modeling and calibration of membership function was involved. Authors also employed Kalman filter to improve the overall performance [14]. Besides fuzzy neutral network based network was applied to robot localization [15] and GPS navigation [16] and performance improvements were verified and quantized. As for the high dynamic problem, Ohlmeyer E.J. proposed a UTC GPS/INS system featured by a bank of prefilters which estimate code delay error and Doppler frequency error for each satellite to relieve the high-dynamic-caused errors. This design was considered more robust to jamming and dynamics [17]. Gao and Lachapelle proposed another new architecture of UTC system with a cooperated tracking loop inside of traditional GPS tracking loops [5]. Effects of Inertial Measurement Unit (IMU) on weak signal tracking were also analyzed. Wang and Li made a research about the positive feedback problem of UTC GPS/INS system and the model of tracking errors was proposed. Besides a track-error estimator was designed to deal with the error positive feedback in UTC systems [8].

Aimed at improving navigation performance in challenging environments, a reinforced and innovative architecture of UTC GPS/INS system is proposed. Firstly a FLAC is applied in the design of adaptive IKF in which the mathematical model of measurement noise is tuned online based on the covariance of innovation sequence. As the empirically determined parameters of MFs have great effects on the adjustments, an optimizing procedure based on neutral network is conducted offline in advance. These techniques result in better estimation for IKF when GPS signal noise changes dramatically. Secondly, a Doppler frequency error estimator for carrier tracking loop, that is, Phase Locked Loop (PLL), is designed to improve high dynamic property of the integrated system. The estimator is based on Kalman filter technology, with carrier phase error of each channel being measurements. The estimated frequency errors are then fed back to carrier tracking loop, making it more robust to high dynamics. Therefore the navigation accuracy, especially velocity accuracy, improves significantly. At last the proposed architecture is assessed by simulations conducted through a self-developed and complete platform. Simulation results demonstrate that the performance improvement is significant for the reinforced and innovative UTC GPS/INS integrated system.

The novel contribution of this paper is that adaptive approaches based on fuzzy logics are adopted to achieve better antijamming performance. A self-developed neutral network is involved to provide an optimization of the parameters of the fuzzy logic adaptive controllers. Besides, a specific Doppler frequency error estimator implemented by Kalman filter is designed to obtain improved performance under high dynamics on foundation of INS-assisted GPS receiver. The proposed fuzzy logic adaptive controllers and Kalman estimators reinforce the GPS/INS integrated navigation system with an improved overall performance under severe jamming and high dynamics.

\section{Problem Description}

For accurate and reliable navigation, PLLs (Phase Locked Loops) of GPS receiver should always be locked in since they can provide much better velocity information for integration navigation. As PLLs suffer most of the noise interference and dynamic stress, DLL (Delay Locked Loop) is generally locked in if PLL is locked in, so most attentions are paid to the vulnerable PLL tracking and correspondingly the sensitive velocity errors in challenging environments. Jamming, changing GPS noise, and high dynamics are the common cases that appear in challenging environments and they are also great threats to accurate and reliable navigation.

For traditional architecture of UTC GPS/INS system which adopts standard extended Kalman filter (EKF) or unscented Kalman filter (UKF) as integration algorithm, the covariance matrix of measurement noise is determined by a priori information and it is never adjusted. For such algorithms problems arise when jamming or interference is involved, for example, strong intended jamming in vast area for military applications, or the multipath interference and low signal-to-noise-ratio (SNR) problem in urban areas for civilian applications. In such cases, the characteristics of GPS signal noise, that is, the measurement noise, vary dramatically, so a constant mathematic model is never able to truly describe the real situations, which will result in poor navigation performance. Hence for accurate and reliable navigation in challenging environments, adaptive IKF which is capable of adjusting the mathematic model of measurement noise is necessary. Fuzzy logic controllers are widely used to implement adaptive IKF based on innovation sequence $[18,19]$. However most of these fuzzy logic controllers are designed depending on personal experience and the essential parameters of membership functions are determined empirically, which with no doubt weakens the effectiveness of fuzzy adaptation. Therefore improvement and optimization for fuzzy logic controllers are needed.

Another serious problem for UTC GPS/INS system is the navigation performance under high dynamics. The errors of aiding information derived from MEMS-based INS become obviously large under high dynamics featured by 
high acceleration and accelerated acceleration. Consequently, the frequency error and phase error of tracking loops are much larger when such dynamics happen. The accumulated phase error is caused by frequency error, which derives from two sources: the first is the error of aiding information due to the error of INS velocity; the second is the error of tracking frequency due to the imperfection of PLL tracking ability. Hence a Doppler frequency error estimator which is capable of estimating the total frequency error of INS-aiding and loop tracking is required. The estimated frequency error should be fed back to the tracking loops so that navigation performance of UTC GPS/INS system can be enhanced in high dynamic scenarios.

\section{Methodology}

3.1. Basic Principles of Adaptive IKF. Thorough research and detailed explanations of standard Kalman filter are provided in many papers [19]. To apply KF for integration, correct state transition matrix and measurement matrix are required as they describe the physical dynamics of the whole system and the mapping relationship between the system states and the measurements. Moreover complete and accurate mathematic models of the process noise and measurement noise are also necessary as they are important factors for optimal estimation and algorithm stability. If any of the information is missing or inaccurate, KF may deduce suboptimal estimates or even get into divergence. Either is unacceptable for accurate and reliable navigation. Much research and calculations were conducted before so that correct state transition matrix and measurement matrix (especially when measurements are pseudorange/pseudorange-rate or inphase/quadrature) for GPS/INS integrated system could be guaranteed. Besides, the stochastic characteristics of the process noise in GPS/INS integrated systems can be precisely measured with method like Allen Variance. The process noise largely refers to white noise and the actuating noise of first-order Markov process of inertial sensor triads, so the statistical characteristics will not vary drastically unless faults or breakdown happens. However situations for measurement noise are quite different. For GPS/INS system, the measurement noise is primarily determined by the noise of GPS outputs (PR and PRR in this paper), and it cannot be sustained at a certain level under various interference conditions. Once measurement noise changes, its mathematical model in IKF, that is, the covariance matrix, needs corresponding corrections to avoid potential filter divergence caused by the inaccuracy of measurement noise model and improve the navigation performance in challenging environments. Several techniques could be considered to accomplish this task and they are categorized into four types: Bayesian, maximum likelihood, correlation (autocorrelation), and covariance matching [18]. The last approach based on innovation variance and implemented by fuzzy logics is chosen in this research.

Innovation (also called residual) is critical information for Kalman filter. It is defined as the difference between real measurements and predicted measurements:

$$
e_{k}=z_{k}-H_{k} x_{k}^{-} \text {. }
$$

In above equation, $\mathbf{k}$ is the time tag and $\mathbf{z}_{\mathbf{k}}$ represents the real measurements. $\mathbf{H}_{\mathbf{k}}$ and $\mathbf{x}_{\mathbf{k}}^{-}$are the measurement matrix and the predicted state vector, respectively. In theory if KF works well, the innovation is zero-mean white noise whose covariance is related to the covariance of estimation and measurement noise, yielding

$$
\mathrm{C}_{\mathrm{e}_{\mathrm{k}}}=\mathbf{H}_{\mathrm{k}} \mathbf{P}_{\mathrm{k}}^{-} \mathbf{H}_{\mathrm{k}}^{\mathrm{T}}+\mathbf{R}_{\mathrm{k}-\mathbf{1}},
$$

where $\mathbf{P}_{\mathbf{k}}^{-}$is predicted covariance matrix of estimation and $\mathbf{R}_{\mathbf{k - 1}}$ is the covariance matrix of measurement noise. $\mathbf{R}_{\mathbf{k}-\mathbf{1}}$ is set as diagonal matrix with the assumption that measurement noises derived from each GPS channel is uncorrelated with each other.

The actual innovation covariance is calculated according to the definition of covariance itself. $N$ latest innovation sequences are multiplied and averaged. The number $N$ is the length of a moving window whose purpose is smoothing. Consider

$$
\mathbf{T}_{\mathbf{e}_{\mathbf{k}}}=\frac{1}{N} \sum_{i=i_{0}}^{k} \mathbf{e}_{\mathbf{i}} \cdot \mathbf{e}_{\mathbf{i}}^{\mathbf{T}} .
$$

In above equation, $i_{0}=k-N+1$ is the start of the moving window.

Generally the number $N$, which is the length of a moving window, is chosen empirically since there is no clear and strict rules to choose $N$. The length of the moving window affects the accuracy and the adjustment speed of the fuzzy logic adaptive controller. The smaller the $N$ is, the faster the controller reacts, but the greater the noise of the actual innovation covariance is. In practical applications, $N$ should be decided by trial and error according to specific conditions.

In normal conditions, the theoretical covariance and real covariance of innovations are identical; otherwise the covariance matrix of measurement noise $\mathbf{R}_{\mathbf{k}-1}$ must be impropriate provided that measurement matrix, state transition matrix, and process noise covariance matrix are all correct and accurate. Hence the fundamental principle of adaptive IKF is to adjust the covariance matrix of measurement noise as what it should be according to the discrepancy between the theoretical covariance and real covariance of innovations; namely,

$$
\Delta \mathbf{D}_{\mathbf{k}}=\operatorname{diagonal}\left(\mathrm{T}_{\mathbf{e}_{\mathrm{k}}}-\mathrm{C}_{\mathrm{e}_{\mathrm{k}}}\right) .
$$

As mentioned above that the measurement noises are assumed to be uncorrelated, the discrepancy matrix $\Delta \mathbf{D}_{\mathbf{k}}$ is also set as a diagonal matrix. Adaptive controllers adjust the covariance matrix of measurement noise as follows:

$$
\mathbf{R}_{\mathrm{k}}=\mathbf{R}_{\mathrm{k}-1}+\Delta \mathbf{R}_{\mathrm{k}},
$$

where $\Delta \mathbf{R}_{\mathbf{k}}$ is the adjustment matrix produced by the adaptive controller according to $\Delta \mathbf{D}_{\mathbf{k}}$.

3.2. Fuzzy Logic Controllers Based on Neutral Network. Fuzzy logic controllers are adopted to implement adaptive IKF. A fuzzy logic controller is a control system based on fuzzy 
TABLE 1: Fuzzy rules of adaptive IKF.

\begin{tabular}{ll}
\hline $\begin{array}{l}\text { If the diagonal element of } \Delta \mathbf{D}_{\mathbf{k}} \\
\text { is }\end{array}$ & $\begin{array}{l}\text { Then the diagonal element of } \\
\Delta \mathbf{R}_{\mathbf{k}} \text { should }\end{array}$ \\
\hline Negative (NT) & Decrease (DC) \\
Zero (ZR) & Maintain (MT) \\
Positive (PT) & Increase (IC) \\
\hline
\end{tabular}

logics, which is a mathematical system that analyzes analog inputs in terms of logical variables and exports outputs for control. The word "fuzzy" means that the logics involved can handle concepts that cannot be expressed as "true" or "false" but rather as "partially true." Fuzzy logic controller is close to human in terms of control procedure, making it a control system that is easy to understand and easy to implement. Fuzzy logic is now widely used in machine control, especially the robust control of very complex systems whose adequate mathematical models are implausible to obtain but control principles are easy to know. The theoretical models of fuzzy logic controllers are mainly depicted by membership functions (MFs) and fuzzy rules. The MFs determine the way that inputs and outputs are mapped to corresponding fuzzy sets. These procedures are called fuzzification (for inputs) and defuzzification (for outputs). The fuzzy rules determine the way that input fuzzy sets are mapped to output fuzzy sets, usually expressed in an "IF-THEN" structure. Therefore, once the inputs and outputs are determined, the implementation of fuzzy logic controller mainly lies in the designs of MFs and fuzzy rules.

As the case of fuzzy adaptive IKF, the input is discrepancy matrix $\Delta \mathbf{D}_{\mathbf{k}}$ and the output is the adjustment matrix $\Delta \mathbf{R}_{\mathbf{k}}$. Fuzzy rules of the adaptive IKF are shown in Table 1. The only task left is the design of membership functions.

Previously the design of MFs depended on personal experience and the essential parameters that shape the MFs are determined empirically. In this paper, a self-defined backpropagation (BP) neutral network is employed to accomplish the optimization for MF parameters. In this feed-forward neutral network, each node performs a particular function (node function) based on inputs and a set of parameters pertaining to this node. The links in this network, however, only serve the purpose of signal transmitting without any weights associated. The $\mathrm{NN}$ used in this paper has 5 layers (3 hidden layers) and it is a single-input-single-output network. The first layer is just an input node without any node function. The second layer has 3 nodes and each node has its specific node function. They also represent the fuzzification membership function: the first as well as last node in second layer has the node function of sigmoid and the second node has the function of gauss:

$$
\begin{gathered}
\operatorname{sigmoid}_{i}(x)=\frac{1}{1+e^{-a_{i}\left(x-b_{i}\right)}} \\
\operatorname{gauss}_{i}(x)=e^{x^{2} / 2 \sigma_{i}^{2}} .
\end{gathered}
$$

For each node the parameters are different and they are to be optimized through network learning. The third layer has 3 nodes too but they share the same node function

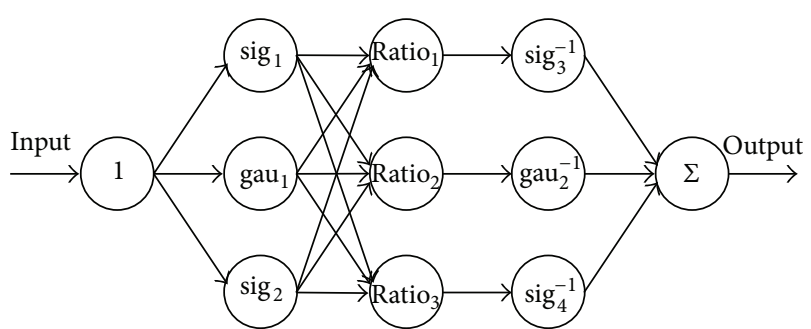

FIGURE 1: The diagram of the self-developed neutral network.

that calculates the ratio of the corresponding 2nd-layer node output to the total 2 nd-layer node outputs:

$$
\operatorname{Ratio}\left(x_{i}\right)=\frac{x_{i}}{\sum_{j=1}^{n} x_{j}}, \quad i=1,2,3, \ldots, n
$$

The fourth layer also has 3 nodes and they represent the inverse of the defuzzification member function: the first and third are the inverse function of sigmoid and the second is the inverse function of gauss. All the function parameters are different, which means 10 parameters in total need optimization. At last the fifth layer has 1 node and its node function is summation. The diagram of the self-defined NN is shown in Figure 1.

One of the most essential properties of BP network is the learning ability provided through back-propagation-based training. This is also the basis for parameter optimization. The specific back-propagation algorithm in this self-defined $\mathrm{NN}$ is different from that of an ordinary BP NN. Here are the details.

Assume that there are $L$ layers in this self-defined $\mathrm{NN}$ and the $k$ th layer has $n(k)$ nodes. The $i$ th node in the $k$ th layer is expressed as $(k, i)$ and the node function as $f_{i}^{k}$. Therefore the output of node $(k, i)$ can be expressed as follows:

$$
O_{i}^{k}=f_{i}^{k}\left(O_{1}^{k-1}, O_{2}^{k-1}, \ldots O_{n(k-1)}^{k-1}, \alpha, \beta, \gamma \ldots\right),
$$

where $\alpha, \beta, \gamma$, and so forth are the node function parameters, which will be adjusted and optimized in back-propagation procedures. These parameters will be in turn used as the parameters of the fuzzy logic controllers.

As this $\mathrm{NN}$ is single-input-single-output network, the error measure (also energy function) is defined as follows:

$$
E=\frac{1}{2}\left(T-O_{1}^{L}\right)^{2},
$$

where $T$ is the expected output while $O_{1}^{L}$ is the output of whole network (the network has $L$ layers and the last layer has only one node). This back-propagation procedure is based on the gradient decent in $E$ over the parameter space.

For the last-layer node the gradient decent in $E$ could be calculated according to (9):

$$
\frac{\partial E}{\partial O_{1}^{L}}=-\left(T-O_{1}^{L}\right)
$$


For each node $(k, i)$ in hidden layers, the chain rule can be applied to calculate

$$
\frac{\partial E}{\partial O_{i}^{k}}=\sum_{m=1}^{n(k+1)} \frac{\partial E}{\partial O_{m}^{k+1}} \frac{\partial O_{m}^{k+1}}{\partial O_{i}^{k}},
$$

where $n(k+1)$ is the number of the nodes in $(k+1)$ th layer and $1 \leq k \leq L-1$. Therefore, the gradient decent in $E$ over parameter $\alpha$ could be obtained:

$$
\frac{\partial E}{\partial \alpha}=\sum_{O^{*} \in S} \frac{\partial E}{\partial O^{*}} \frac{\partial O^{*}}{\partial \alpha} .
$$

In above equation, $S$ represents the set of nodes whose outputs $O^{*}$ are related to parameter $\alpha$.

Correspondingly the correction for parameter $\alpha$ is

$$
\Delta \alpha=-\eta \frac{\partial E}{\partial \alpha},
$$

where $\eta$ is the learn rate which can be expressed as

$$
\eta=\frac{p}{\sqrt{\sum_{\alpha}(\partial E / \partial \alpha)^{2}}},
$$

$p$ is the step size of the gradient decent.

With back-propagation algorithm and training data, the parameter optimization could be achieved and results will be shown in Section 4.

It is worth noting that the training procedure has great influence on the performance of the BP neutral network. In this paper we guarantee the offline-trained parameters to be good enough for online application through two things. Firstly and basically, the principles of the fuzzy logic adaptive controllers are the same. The adaptive controllers work in totally the same way no matter online or offline, which makes the offline-trained parameters being used online possible. Secondly, the characteristics of the training data are the same as that applied in the simulations, which will be introduced in Section 4. They are both gauss white noise and the only difference lies on the standard deviation of the noise. However in practical applications it is best to obtain training data during prerun in real circumstances and finish the parameter optimization in this phase.

3.3. Doppler Frequency Error Estimator for PLL. The most important feature of UTC GPS/INS system is the INS-assisted GPS receiver which has a much wider effective bandwidth than stand-alone receiver. This feedback from INS to GPS receiver gives the UTC integrated system a much better high dynamic property than loosely or tightly couple system. However the INS navigation error will obviously increase during high dynamics when low-end MEMS-based IMU is used, so the availability and effectiveness of INS-aiding information drop, especially for the vulnerable PLL. Therefore some other techniques have to be adopted to guarantee accurate and reliable navigation during high dynamics.

Figure 2 illustrates a model of PLL aided by INS, and the symbol " $s$ " represents the operator of Laplace Transform. The PLL phase error can be derived as

$$
\delta \varphi=\varphi_{d}+\varphi_{c}+\varphi_{w}-\varphi_{l} .
$$

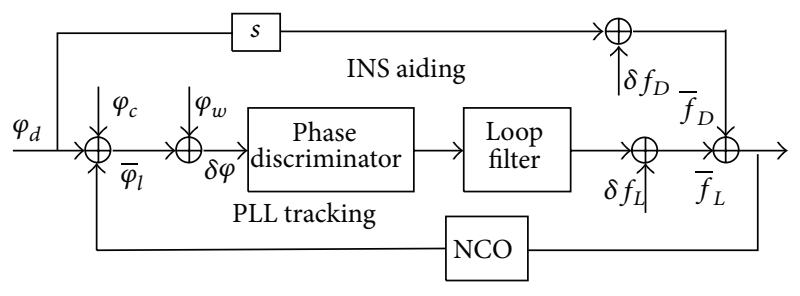

Figure 2: Model of PLL aided by INS.

In the equation, $\varphi_{d}$ is the phase change caused by Doppler shifting and $\varphi_{c}$ represents correlated errors such as oscillator instabilities of the GPS receiver and satellite and so forth. $\varphi_{w}$ is the wide band thermal noise and $\varphi_{l}$ denotes the local carrier phase generated by Numerical Controlled Oscillator (NCO). Apart from the white noise, two components contribute to the frequency error: the aiding error $\delta f_{D}$ due to the erroneous INS velocity and tracking error $\delta f_{L}$ due to the limitation of PLL tracking ability. So the rate of carrier phase error can be expressed as follows:

$$
\delta \dot{\varphi}=2 \pi \delta f_{\mathrm{PLL}}=2 \pi\left(\delta f_{D}+\delta f_{L}\right),
$$

where $\delta f_{\text {PLL }}$ is the PLL frequency error. The relationship between $\delta f_{D}$ and aiding velocity error $\boldsymbol{\delta} \mathbf{V}$ can be expressed as $[8]$

$$
\delta f_{D}=\frac{(\boldsymbol{\delta} \mathbf{V} \cdot \mathbf{L}) f_{L}}{c},
$$

where $\mathbf{L}$ is the Line of Sight (LOS) vector between GPS receiver and a certain satellite.

According to the analysis above, a Doppler frequency error estimator for PLL is established based on Kalman filter technology. The state vector consists of PLL phase error and frequency error for each GPS channel, so it is expressed as

$$
\mathbf{X}_{\mathbf{f}}=\left[\delta \varphi_{1}, \delta \varphi_{2}, \ldots, \delta \varphi_{m}, \delta f_{\mathrm{PLL} 1}, \delta f_{\mathrm{PLL} 2}, \ldots, \delta f_{\mathrm{PLL} m}\right]^{\mathrm{T}},
$$

where $\delta \varphi_{i}$ and $\delta f_{\mathrm{PLL} i}(i=1,2, \ldots, m)$ are the phase error and frequency error of the acquired satellite $i$, respectively. The dimension of $\mathbf{X}_{\mathbf{f}}$ is determined by the number of available satellites, noted by $m$.

States transition matrix $\mathbf{F}_{\mathrm{f}}$ can be easily derived according to (15):

$$
\mathbf{F}_{\mathbf{f}}=\left[\begin{array}{cc}
0_{m \times m} & 2 \pi \mathbf{E}_{m \times m} \\
0_{m \times m} & 0_{m \times m}
\end{array}\right],
$$

where $\mathbf{E}_{m \times m}$ is an $m \times m$ unit matrix.

Process noise vector $\mathbf{W}_{\mathbf{f}}$ contains frequency noise of each channel and they are assumed to be uncorrelated white noise:

$$
\mathbf{W}_{\mathbf{f}}=\left[w_{f_{\mathrm{PLL1}}}, w_{f_{\mathrm{PLL} 2}} \ldots w_{f_{\mathrm{PLL} m}}\right]^{\mathrm{T}} .
$$

Therefore the process noise matrix $\mathbf{G}_{\mathbf{f}}$ complies with the following equation:

$$
\mathbf{G}_{\mathbf{f}}=\left[\begin{array}{ll}
0_{m \times m} & 0_{m \times m} \\
0_{m \times m} & \mathbf{E}_{m \times m}
\end{array}\right] .
$$


The measurements of the Doppler frequency error estimator are the phase errors provided by PLL discriminator of each channel, so the measurement matrix is

$$
\mathbf{H}_{\mathbf{f}}=\left[\begin{array}{ll}
\mathbf{E}_{m \times m} & 0_{m \times m}
\end{array}\right] .
$$

Discretization has to be conducted on these timecontinuous models and then the Doppler frequency errors of PLL could be estimated at each update. These estimates are fed back to compensate for PLL tracking frequency errors, improving tracking ability directly and sufficiently. This leads to significant improvement of PRR accuracy, especially under high dynamic conditions.

3.4. Integrated Kalman Filter. For the integrated Kalman filter, the filter states consist of position errors, velocity errors, attitude errors, gyroscope biases, and accelerometer biases, 15 elements in total:

$$
\begin{aligned}
& \mathbf{X}=[\text { Position Errors (3), } \\
& \text { Velocity Errors (3), Attitude Errors (3), }
\end{aligned}
$$$$
\text { Gyroscope Bias (3)...Accelerometer Bias (3) }]^{\mathrm{T}} \text {. }
$$

The numbers in brackets represent the number of elements of each state set. Extended Kalman filter (EKF) is adopted to estimate the states above. Position errors, velocity errors, and attitude errors are used to correct INS navigation results.

As a fuzzy adaptive controller is involved, the mathematic model of EKF is distinguished from traditional ones. Basically it can be implemented by two steps.

Step 1. The algorithms of traditional EKF are

$$
\begin{aligned}
& \mathbf{K}_{\mathbf{k}}=\mathbf{P}_{\mathbf{k}}^{-} \mathbf{H}_{k}^{\mathrm{T}}\left(\mathbf{H}_{\mathbf{k}} \mathbf{P}_{\mathbf{k}}^{-} \mathbf{H}_{\mathbf{k}}^{\mathrm{T}}+\mathbf{R}_{\mathbf{k}}\right)^{-1} \\
& e_{k}=z_{k}-H_{k} x_{k}^{-} \\
& \mathbf{x}_{\mathrm{k}}=\mathbf{x}_{\mathrm{k}}^{-}+\mathrm{K}_{\mathrm{k}} \mathbf{e}_{\mathrm{k}} \\
& \mathrm{x}_{\mathrm{k}+1}^{-}=\Phi_{\mathrm{k}} \mathrm{x}_{\mathrm{k}} \\
& \mathbf{P}_{\mathbf{k}}=\left(\mathbf{I}-\mathbf{K}_{\mathbf{k}} \mathbf{H}_{\mathbf{k}}\right) \mathbf{P}_{\mathbf{k}}^{-} \\
& P_{k+1}^{-}=F_{k+1 / k} P_{k} F_{k+1 / k}^{T}+Q_{k} \text {. }
\end{aligned}
$$

In equations above, the representations of the mathematical notations that are not explained in this paper could be referred to [19]. Since the filter model has been fully developed in previous research, the expression of each matrix and other details are saved here.
Step 2. The adaptation of measurement covariance matrix is

$$
\begin{gathered}
\mathbf{C}_{\mathbf{e}_{\mathbf{k}}}=\mathbf{H}_{\mathbf{k}} \mathbf{P}_{\mathbf{k}}^{-} \mathbf{H}_{\mathbf{k}}^{\mathrm{T}}+\mathbf{R}_{\mathbf{k}} \\
\mathbf{T}_{\mathbf{e}_{\mathrm{k}}}=\frac{1}{\mathbf{N}_{\mathrm{i}=\mathbf{i}_{0}}} \sum_{\mathbf{i}}^{\mathbf{k}} \mathbf{e}_{\mathrm{i}}^{\mathrm{T}} \\
\Delta \mathbf{D}_{\mathbf{k}}=\text { diagonal }\left(\mathbf{T}_{\mathbf{e}_{\mathbf{k}}}-\mathbf{C}_{\mathbf{e}_{\mathbf{k}}}\right) \\
\Delta \mathbf{R}_{\mathbf{k}}=\operatorname{FLAC}\left(\Delta \mathbf{D}_{\mathbf{k}}\right) \\
\mathbf{R}_{\mathbf{k}+\mathbf{1}}=\mathbf{R}_{\mathbf{k}}+\Delta \mathbf{R}_{\mathbf{k}} .
\end{gathered}
$$

In equations above, the representations of the mathematical notations could be referred to in Section 3.1 of this paper. After the adaptation of measurement covariance matrix, algorithms of EKF will be conducted again with the new measurement covariance matrix.

Summarizing all the innovative features explained in Sections 3.1 to 3.4 , the scheme diagram of the reinforced UTC GPS/INS system is shown in Figure 3. The blocks in the boxes with dashed line represent the techniques that distinguish the reinforced and innovative architecture from a standard or traditional one. The symbols $\rho$ and $\dot{\rho}$ represent the pseudorange and pseudorange rate, respectively.

\section{Simulation and Analysis}

4.1. Simulation Configurations. To verify the effectiveness of the proposed architecture, a whole self-developed simulation package is employed and its compositions are shown in Figure 4 . Based on information provided by Trajectory Simulator and GPS Constellation Simulator, IF data (Intermediate Frequency data, the digital data after down-convert) and IMU data are generated and they are used by GPS Software Defined Receiver (SDR) and INS, respectively. IKF fuses the information from both subsystems and implements their mutual assistance. The three blocks in the red-dashed-line box are the reinforced UTC GPS/INS system, which has been described in detail in Figure 3. More details about this simulation package could be referred to in [20].

The 3-dimensional position of the 89-second simulated trajectory is shown in Figure 5(a) and the magnitudes of velocity and acceleration are shown in Figure 5(b). Simulated vehicle is static at the beginning and accelerates to $300 \mathrm{~m} / \mathrm{s}$ during $2 s-5 s$, which results in the first peak of acceleration plot. Moreover, a pitch maneuver which results in huge acceleration up to $1130 \mathrm{~m} / \mathrm{s}^{2}$ is conducted during $73 \mathrm{~s}-75 \mathrm{~s}$ (the enlarged view of acceleration is plotted in the lower left of Figure 5(b)). The huge acceleration is employed to evaluate the performance of UTC GPS/INS system under high dynamics.

The single-frequency $(\mathrm{L} 1,1575.42 \mathrm{MHz})$ GPS SDR uses a five-satellite constellation with a GDOP of 1.85 and PR/PRR update rate is $1 \mathrm{kHz}$. Typical second-order tracking loops are applied for both DLL and PLL, whose bandwidths are $0.1 \mathrm{~Hz}$ and $2 \mathrm{~Hz}$, respectively. The error characteristics of IMU are basically determined in accordance with generic MEMS 


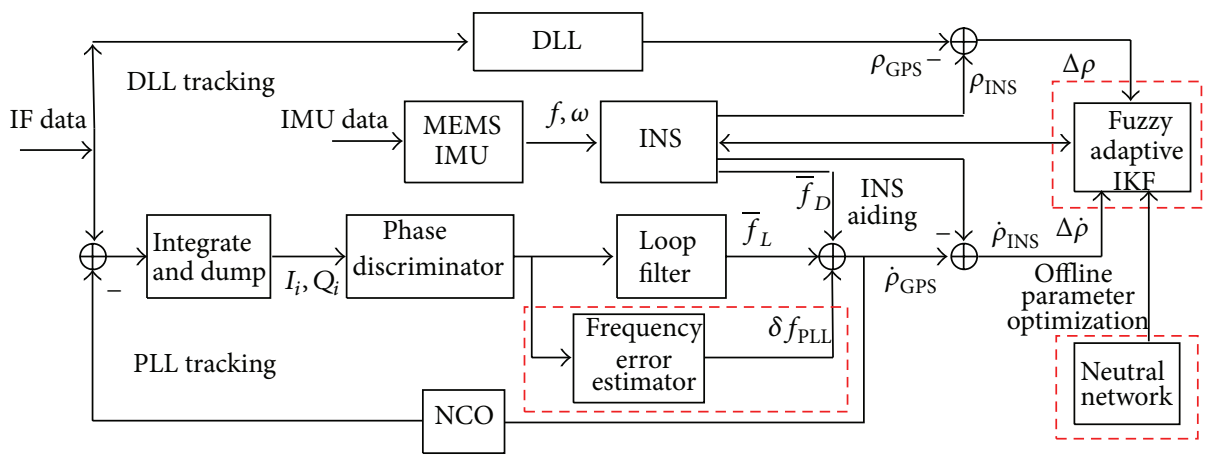

FIGURE 3: Scheme diagram of the reinforced UTC GPS/INS system.

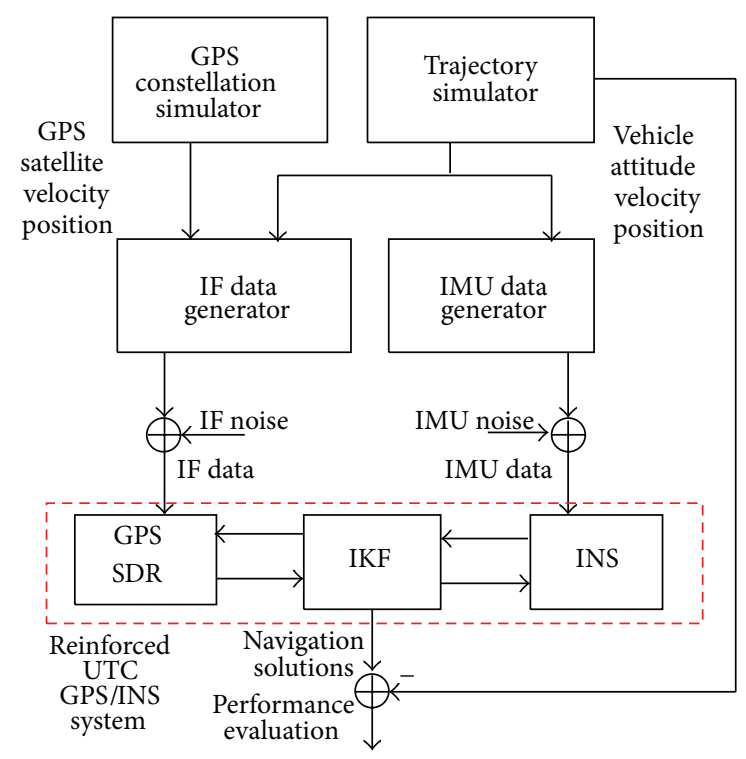

FIGURE 4: Diagram of simulation package.

TABLE 2: IMU error characteristics.

\begin{tabular}{lcc}
\hline Error sources & \multicolumn{2}{c}{ Error values } \\
& Gyroscopes & Accelerometers \\
\hline Bias & $10 \mathrm{deg} / \mathrm{h}$ & $3 \mathrm{mg}$ \\
Scale factor & $100 \mathrm{ppm}$ & $100 \mathrm{ppm}$ \\
Random walk & $0.05 \mathrm{deg} / \sqrt{\mathrm{h}}$ & $0.05 \mathrm{~m} / \mathrm{s} / \sqrt{\mathrm{h}}$ \\
\hline
\end{tabular}

sensors and they are listed in Table 2. The IMU data rate is $1 \mathrm{kHz}$ while the KF update rate is $10 \mathrm{~Hz}$. Navigation results are provided in Geographic Coordinates (East-North-Up). As each satellite channel is independent and equivalent, only the loop tracking results and PRR of satellite \#5 (pseudorandom number 5) are displayed in the following section.

4.2. Assessment of Neutral-Network-Based Fuzzy Adaptive $I K F$. As mentioned in Section 3.2, the membership functions for fuzzification and defuzzification are chosen (sigmoid and gauss) but their parameters are to be optimized. Sigmoid function is chosen for input linguistic words Negative
(NG), Positive (PG) and output linguistic words Decrease (DC), Increase (IC) while gauss function is chosen for input linguistic word Zero (ZR) and output linguistic word Maintain (MT). Each sigmoid function has two parameters and gauss has one, so in total there are 10 parameters to be optimized. Optimization procedure based on the backpropagation algorithm detailed in Section 3.2 is implemented through Matlab. 30000 randomly generated training samples are used to train the neutral network to obtain the optimized parameters and results are shown in Figure 6(a). At first a set of parameters are empirically chosen to start the training. With the training proceeding, all the parameters converge to optimized values. MFs based on empirical and optimized parameters are listed in Table 3. The last plot of Figure 6(a) shows the decrease in training error of the neutral network. The comparisons between empirical and optimized MFs are depicted in Figure 6(b).

To verify the effectiveness of neutral-network-based fuzzy adaptive IKF, comparative integrated navigation simulations of UTC GPS/INS system are conducted under three different conditions: standard IKF without any adaptation ("Standard" in legends), fuzzy adaptive IKF without NN-based optimization ("FLAC without NN" in legends), and fuzzy adaptive IKF with NN-based optimization ("FLAC with NN" in legends). During $20 \mathrm{~s}-40 \mathrm{~s}$, interference is simulated so that the pseudorange rate noise (i.e., the measurement noise) increases from $0.2 \mathrm{~m} / \mathrm{s}$ to $2 \mathrm{~m} / \mathrm{s}$ (both in terms of standard deviation). During other periods, the noise is $0.2 \mathrm{~m} / \mathrm{s}$. At first for all the three different systems the PRR measurement noise models, that is, measurement noise covariance matrices, are $0.2 \mathrm{~m} / \mathrm{s}$, fitting the real situation well, so the ratios of actual and theoretical innovation variance for all integrated systems are around $1(0 \mathrm{~dB})$. However during $20 \mathrm{~s}-40 \mathrm{~s}$, as the measurement noise increases remarkably, the unchanged model that standard IKF adopts (shown in the upper plot of Figure 6(c) by blue dot-dashed line) fails to fit with reality, so the ratio of actual and theoretical innovation variance increases correspondingly from nearly $1(0 \mathrm{~dB})$ up to 100 $(20 \mathrm{~dB})$. On the contrary, IKFs with fuzzy logic adaptive controllers (FLACs) are capable of adjusting the measurement noise model to $2 \mathrm{~m} / \mathrm{s}$ online, which results in the ratio of actual and theoretical innovation variance stays around $1(0 \mathrm{~dB})$. Moreover, the FLAC with NN-based optimization 


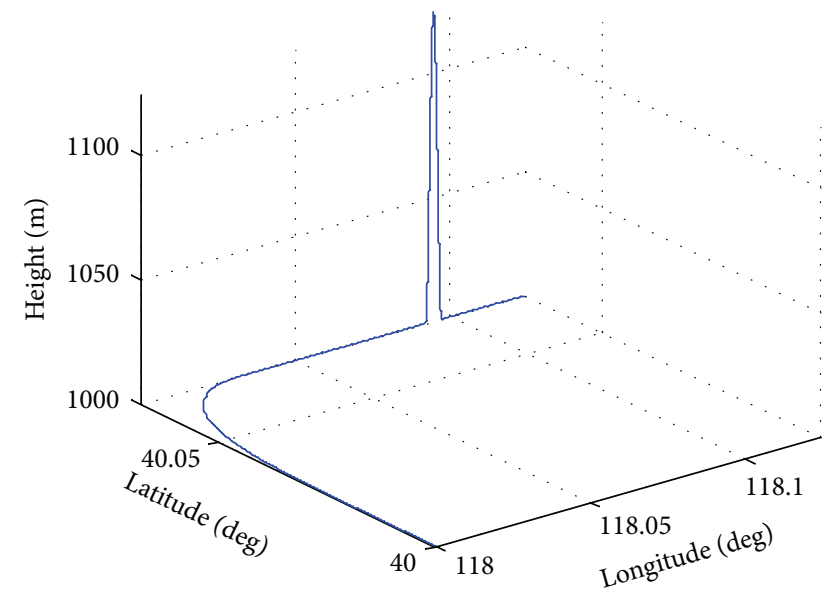

(a) Three-dimensional position
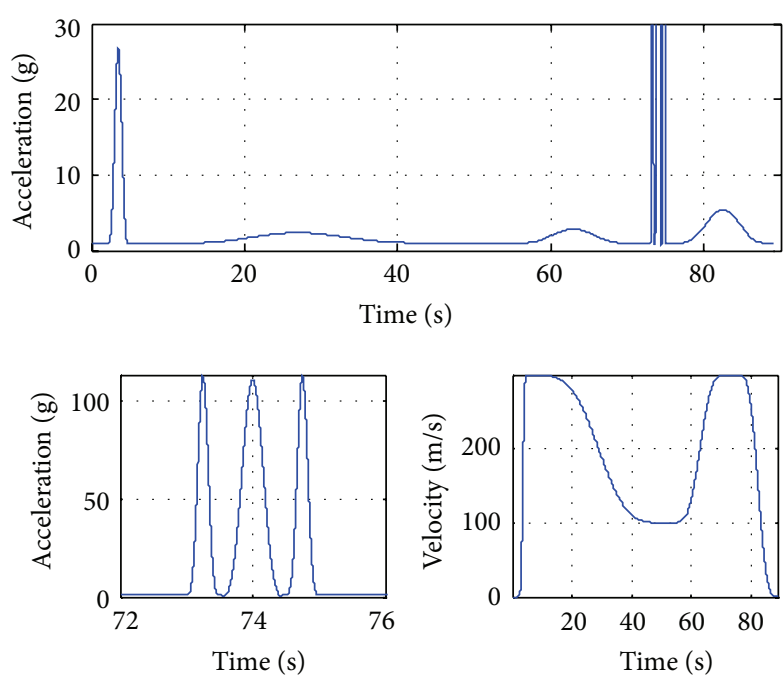

(b) Acceleration and velocity

FIGURE 5: Simulated trajectory.

TABLE 3: MFs based on empirical and optimized parameters.

\begin{tabular}{lcccc}
\hline I/O & Type & & Membership functions & \\
\hline & & Negative (NT) & Zero (ZR) & Positive (PT) \\
Input & Empirical & $\frac{1}{1+e^{10(x+0.6)}}$ & $e^{x^{2} /\left(2 \times 0.3^{2}\right)}$ & $\frac{1}{1+e^{-10(x-0.6)}}$ \\
& Optimized & $\frac{1}{1+e^{10.01(x+0.603)}}$ & $e^{x^{2} /\left(2 \times 0.941^{2}\right)}$ & $\frac{1}{1+e^{-9.99(x-0.603)}}$ \\
& & Decrease (DC) & Maintain (MT) & $\frac{1}{1+e^{-9(x-0.8)}}$ \\
Output & Empirical & $\frac{1}{1+e^{9(x+0.8)}}$ & $e^{x^{2} /\left(2 \times 0.3^{2}\right)}$ & $\frac{1}{1+e^{-9.99(x-0.603)}}$ \\
& Optimized & $\frac{1}{1+e^{10(x+0.603)}}$ & $e^{x^{2} /\left(2 \times 0.941^{2}\right)}$ & \\
\hline
\end{tabular}

makes a better adaptation. Firstly, the fluctuations of the parameters of the noise model are largely suppressed and secondly the transient errors of the ratios at $20 \mathrm{~s}$ and $40 \mathrm{~s}$ are much smaller, as shown in Figure 6(c). It is worth noting that as a moving window of 20 is employed when calculating real innovation variance as (3) implies, the adjustment of measurement noise model is lagged to some extent, especially when the added noise disappears at the 40th second. At last the velocity errors are compared among these three systems and shown in Figure 6(d). The incremental improvements provided by fuzzy logic adaptive IKF and neutral-networkbased parameter optimization, respectively, under challenging environments are quite obvious. The standard deviation of total velocity during $20 \mathrm{~s}-40 \mathrm{~s}$ is $0.121 \mathrm{~m} / \mathrm{s}$ for standard IKF while $0.054 \mathrm{~m} / \mathrm{s}$ for fuzzy adaptive IKF and $0.036 \mathrm{~m} / \mathrm{s}$ for fuzzy adaptive IKF with NN-based optimization.

4.3. Assessment of Doppler Frequency Error Estimator. As mentioned in Section 4.1, a drastic pitch maneuver is scheduled during $73 \mathrm{~s}$ to $75 \mathrm{~s}$ to assess the frequency error estimator in high dynamics. The PLL is a traditional second-order closed loop that is sensitive to acceleration, so when the pitch maneuver that results in huge acceleration is conducted,
PLL tracking phase error will become obviously greater although the total velocity is stable. The output of the Doppler frequency error estimator for satellite \#5 is shown in Figure $7(\mathrm{a})$, along with vehicle's acceleration on vertical axis which contributes to the most of the total acceleration. As the magnitude of acceleration increases, the magnitude of frequency error increases, up to about $10 \mathrm{~Hz}$. In standard architecture, PLL loses lock because the bandwidth $(2 \mathrm{~Hz})$ is not wide enough. However for the innovative scheme, frequency error estimator compensates for most of the frequency error, so it further improves the PLL tracking ability on the basis of INS aiding under high dynamics.

The phase error and PRR error during $72 \mathrm{~s}$ to $76 \mathrm{~s}$ are shown in Figure 7(b) for both innovative and standard architectures. For the standard one, the phase error not only becomes much larger but also changes rapidly, because the huge acceleration forces the PLL to lose track and cycle-slip occurs frequently. Consequently PRR error gets worse, up to $3.02 \mathrm{~m} / \mathrm{s}$, and it changes quickly, too. In contrast, the phase error of innovative architecture is restrained within $0.48 \mathrm{rad}$ (27.5 degrees) and cycle-slip never occurs. The PRR accuracy is better than $1 \mathrm{~m} / \mathrm{s}$ most of the time. The comparative navigation performances of the two schemes shown in Figure 7(c) 

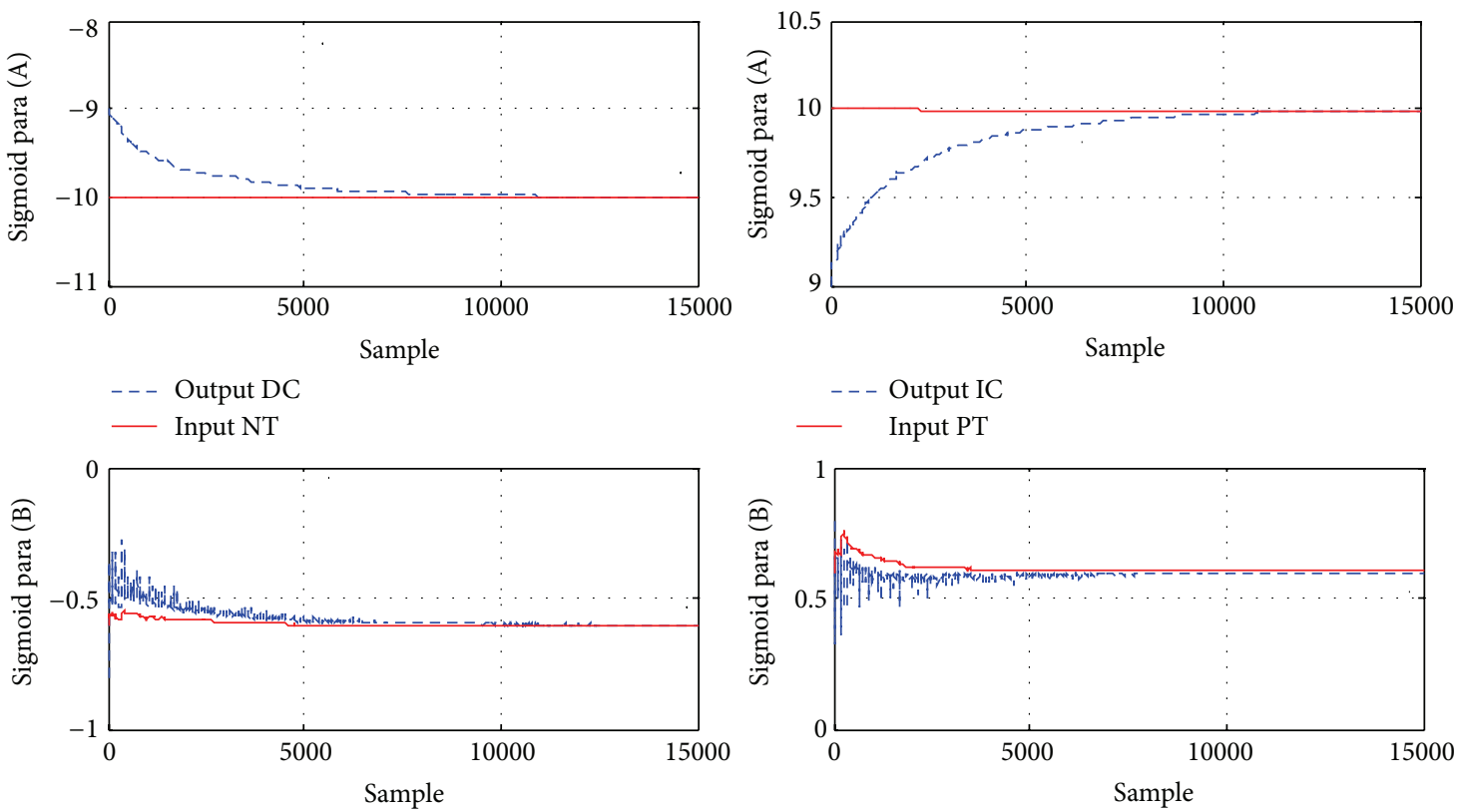

- - - Output IC

- Input PT

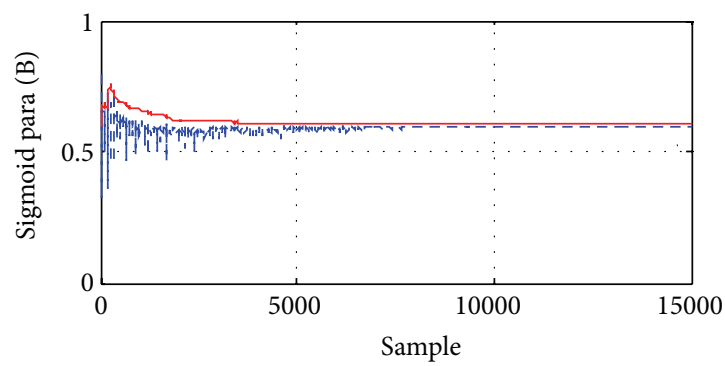

--- Output DC

- Input NT

--- Output IC

— Input PT
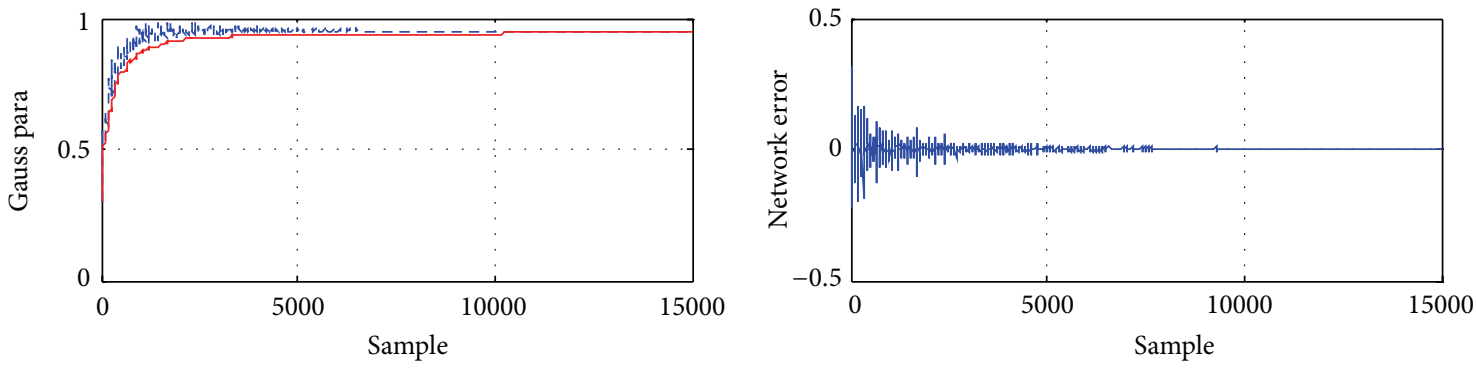

- - Output MT

— Input ZR

(a) Parameters optimization results
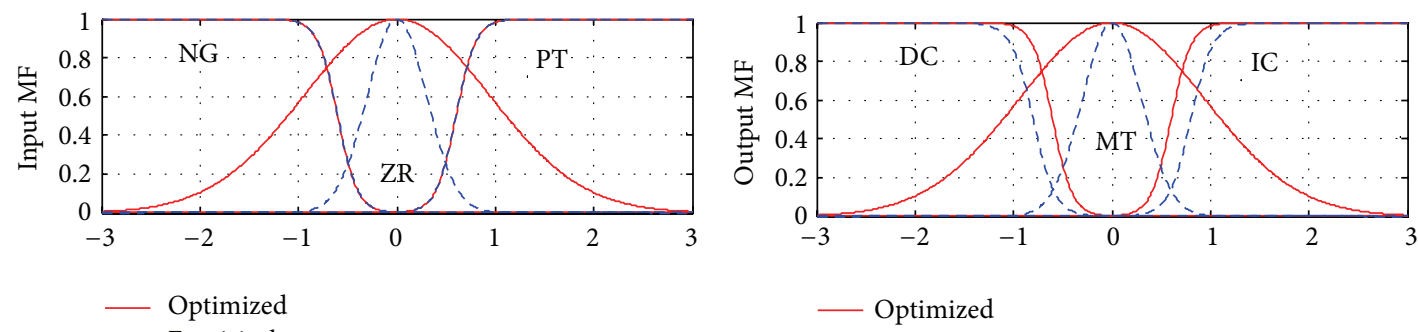

- Optimized

- - Empirical

- - - Empirical

(b) MFs based on optimized and empirical parameters
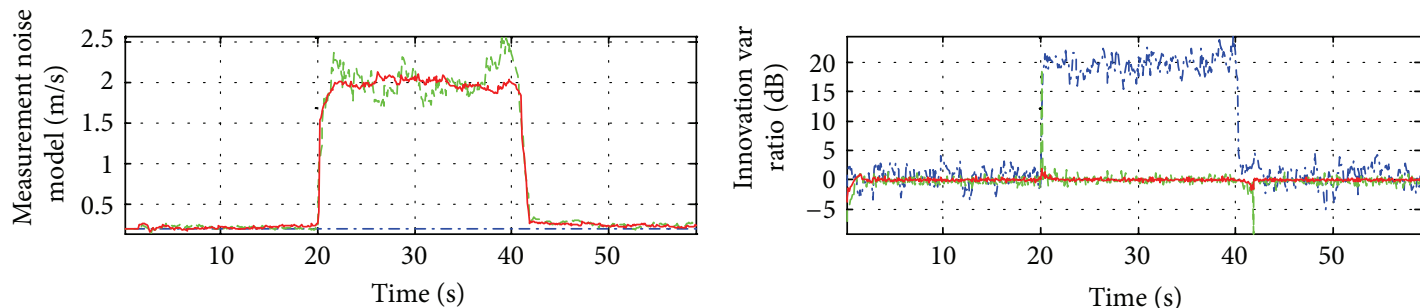

$\cdot-\cdot-$ Standard

— FLAC with NN

- - FLAC without NN

-.- Standard — FLAC with NN

--- FLAC without NN

(c) Performance of FLACs and standard IKFs

Figure 6: Continued. 

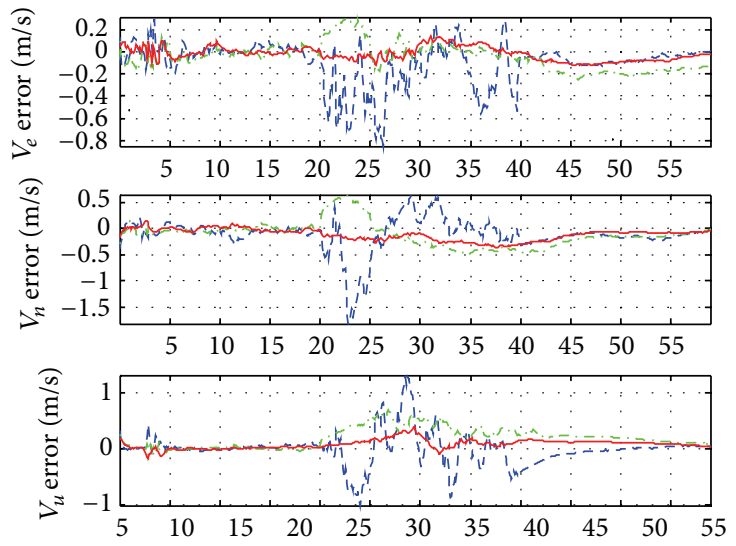

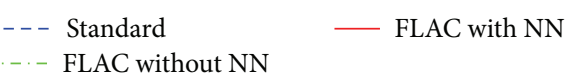

(d) Velocity error comparisons (0-60 s)

FIGURE 6: Comparative results for neutral-network-based fuzzy adaptive IKF.

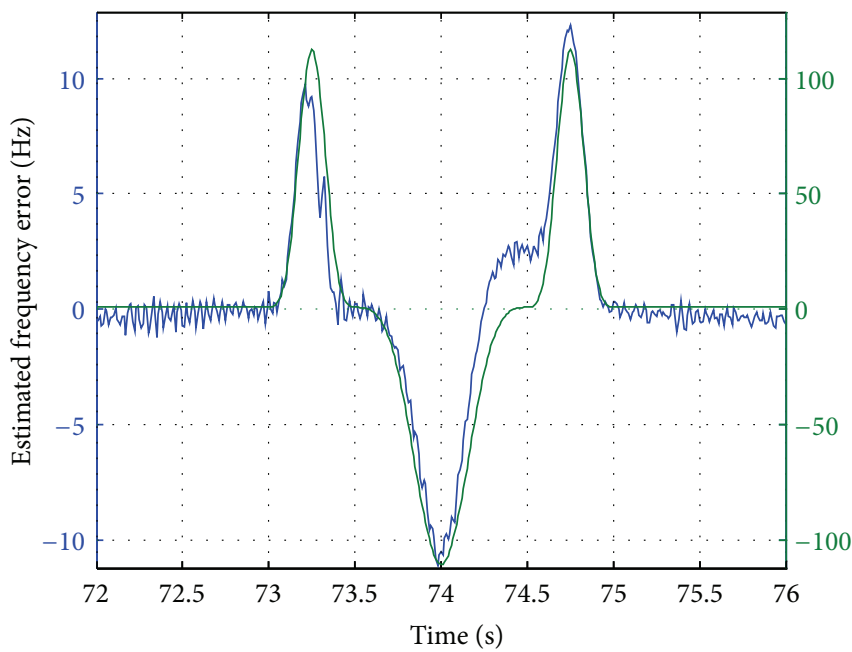

(a) Estimated PLL frequency error

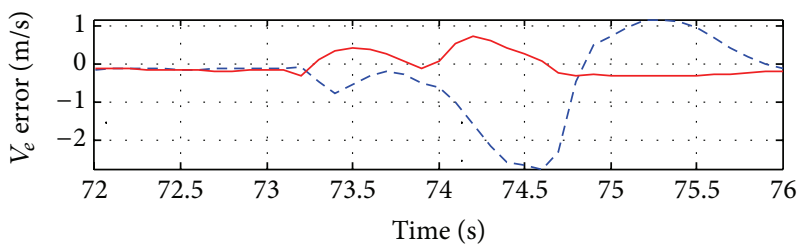

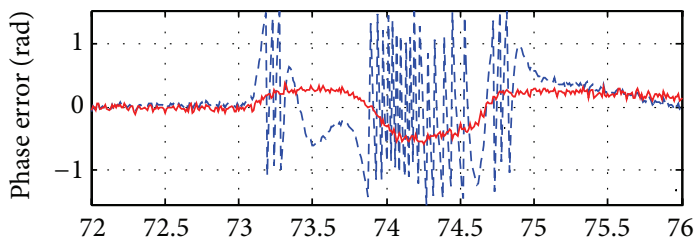

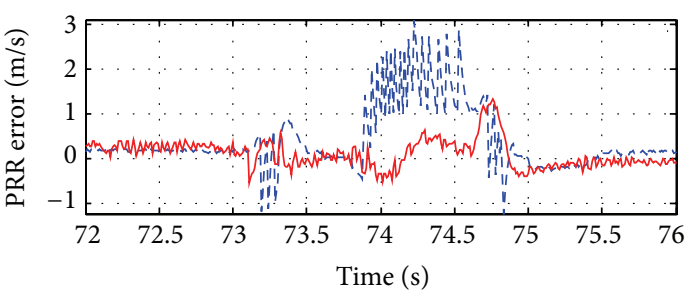

- - - Standard

— Innovative

(b) Phase and PRR error comparisons

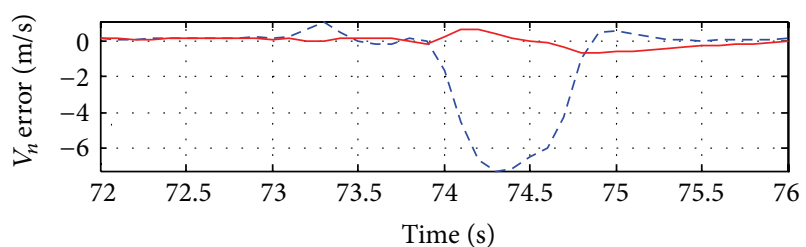

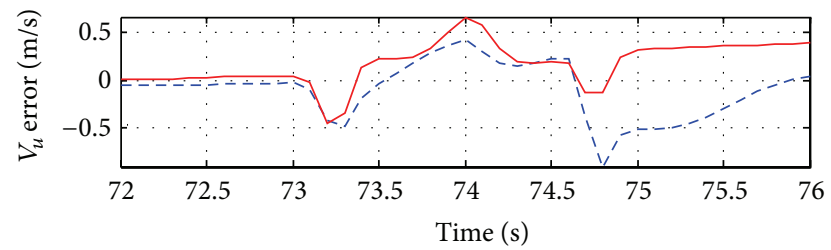

- - Standard

- Innovative

(c) Velocity error comparisons (72s-76s)

FIGURE 7: Comparative results for frequency error estimator. 
reveal that the enhancement provided by Doppler frequency error estimator in high dynamics is remarkable. In terms of maximum velocity error, the improved velocity accuracy is $1.32 \mathrm{~m} / \mathrm{s}$ during $72 \mathrm{~s}-76 \mathrm{~s}$ while the standard system just makes it to $7.91 \mathrm{~m} / \mathrm{s}$. In terms of velocity standard deviation, the proposed and traditional schemes' performances are $0.22 \mathrm{~m} / \mathrm{s}$ and $2.24 \mathrm{~m} / \mathrm{s}$, respectively, during the same period.

\section{Conclusion}

In this paper, a reinforced UTC GPS/INS integrated system is proposed for performance improvement under challenging environments. The innovative architectural features include neutral-network-based fuzzy logic adaptive IKF and a Doppler frequency error estimator for PLL. The fuzzy logic controllers play a key role in performance improvement under interference, jamming, or changing GPS noise conditions. The measurement noise covariance matrix of IKF is corrected online through FLAC according to innovation covariance. Moreover an optimization algorithm for parameters of membership functions based on a self-developed BP neutral network is proposed and detailed. On the other hand, the Doppler frequency error estimator which is based on Kalman filter technology focuses on problem of PLL loosing lock under high dynamics. The frequency error of each channel is estimated according to the phase error. Through the feedback of the estimated frequency errors, PLL tracking ability is remarkably reinforced. All the algorithms are derived and the estimator is fully established. Finally, a whole simulation platform is employed to verify the effectiveness of the proposed techniques. Results presented in this paper demonstrate that the reinforced UTC GPS/INS integrated system improves the navigation performance significantly under challenging environments compared with that of standard system and therefore it is a more advanced solution to accurate and reliable navigation applications.

\section{Conflict of Interests}

The authors declare that there is no conflict of interests regarding the publication of this paper.

\section{Acknowledgment}

This research is supported and funded by the Graduate Innovative Practice Foundation of Beihang University (no. YCSJ-01-201409). The authors would appreciate the support and fund.

\section{References}

[1] A. Noureldin, A. El-Shafie, and N. El-Sheimy, "Adaptive neurofuzzy module for inertial navigation system/global positioning system integration utilising position and velocity updates with real-time cross-validation," IET Radar, Sonar and Navigation, vol. 1, no. 5, pp. 388-396, 2007.

[2] S.-B. Kim, J.-C. Bazin, H.-K. Lee, K.-H. Choi, and S.-Y. Park, "Ground vehicle navigation in harsh urban conditions by integrating inertial navigation system, global positioning system, odometer and vision data," IET Radar, Sonar and Navigation, vol. 5, no. 8, pp. 814-823, 2011.

[3] Y. Yang, D.-J. Feng, W.-M. Zhang, X.-S. Wang, and S.-P. Xiao, "Detection of chaff centroid jamming aided by GPS/INS," IET Radar, Sonar and Navigation, vol. 7, no. 2, pp. 130-142, 2013.

[4] M. Lashley, D. M. Bevly, and J. Y. Hung, "Analysis of deeply integrated and tightly coupled architectures," in Proceedings of the IEEE/ION Position, Location and Navigation Symposium (PLANS '10), pp. 382-396, Indian Wells, Calif, USA, May 2010.

[5] G. Gao and G. Lachapelle, "A novel architecture for ultra-tight HSGPS-INS integration," Journal of Global Positioning Systems, vol. 7, pp. 46-61, 2008.

[6] G. Egziabher, "GNSS Solutions: weighting GNSS observations and variations of GNSS/INS integration," in Global Navigation Satellite Systems (GNSS '07), pp. 28-33, Springer, 2007.

[7] P. D. Groves and D. C. Long, "Combating GNSS interference with advanced inertial integration," Journal of Navigation, vol. 58, no. 3, pp. 419-432, 2005.

[8] X.-L. Wang and Y.-F. Li, "An innovative scheme for SINS/GPS ultra-tight integration system with low-grade IMU," Aerospace Science and Technology, vol. 23, no. 1, pp. 452-460, 2012.

[9] D.-J. Jwo, C.-F. Yang, C.-H. Chuang, and T.-Y. Lee, "Performance enhancement for ultra-tight GPS/INS integration using a fuzzy adaptive strong tracking unscented Kalman filter," Nonlinear Dynamics, vol. 73, no. 1-2, pp. 377-395, 2013.

[10] C. Hide, T. Moore, and M. Smith, "Adaptive Kalman filtering for low-cost INS/GPS," Journal of Navigation, vol. 56, no. 1, pp. 143-152, 2003.

[11] D. Loebis, R. Sutton, J. Chudley, and W. Naeem, "Adaptive tuning of a Kalman filter via fuzzy logic for an intelligent AUV navigation system," Control Engineering Practice, vol. 12, pp. 1531-1539, 2004.

[12] H. S. Son, J. B. Park, and Y. H. Joo, "Fuzzy c-means clusteringbased smart tracking model for three-dimensional manoeuvring target including unknown acceleration input," IET Radar, Sonar and Navigation, vol. 7, no. 6, pp. 623-634, 2013.

[13] S. Y. Noh, J. B. Park, and Y. H. Joo, "Intelligent tracking algorithm for manoeuvering target using Kalman filter with fuzzy gain," IET Radar, Sonar and Navigation, vol. 1, no. 3, pp. 241-247, 2007.

[14] C.-T. Sun and J.-S. Jang, "Fuzzy modeling based on generalized neural networks and fuzzy clustering objective functions," in Proceedings of the 30th IEEE Conference on Decision and Control, pp. 2924-2929, Brightonm, UK, December 1991.

[15] T. T. van Nguyen, M. D. Phung, T. H. Tran, and Q. V. Tran, "Mobile robot localization using fuzzy neural network based extended Kalman filter," in Proceedings of the IEEE International Conference on Control System, Computing and Engineering (ICCSCE '12), pp. 416-421, Penang, Malaysia, November 2012.

[16] D.-J. Jwo and H.-C. Huang, "GPS navigation using fuzzy neural network aided adaptive extended Kalman filter," in Proceedings of the 44th IEEE Conference on Decision and Control, pp. 78407845, Seville, Spain, December 2005.

[17] E. J. Ohlmeyer, "Analysis of an ultra-tightly coupled GPS/INS system in jamming," in Proceedings of the IEEE/ION Position, Location, and Navigation Symposium, pp. 44-53, Coronado, Calif, USA, April 2006.

[18] J. Ali, "Strapdown inertial navigation system/astronavigation system data synthesis using innovation-based fuzzy adaptive Kalman filtering," IET Science, Measurement and Technology, vol. 4, no. 5, pp. 246-255, 2010. 
[19] M. Liu and F. Xiong, "A fuzzy adaptive GPS/INS integrated navigation algorithm," in Proceedings of the International Conference on Advanced in Control Engineering and Information Science (CEIS '11), pp. 660-664, August 2011.

[20] X. Wang, J. Zhang, W. Wang, and P. Gao, "An innovative architecture of UTC GPS/INS system with improved performance under severe jamming," Discrete Dynamics in Nature and Society, vol. 2014, Article ID 185618, 8 pages, 2014. 


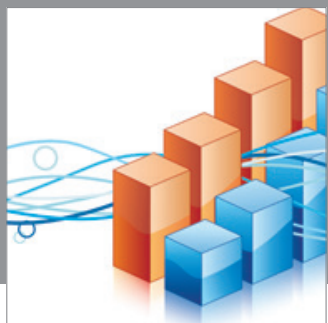

Advances in

Operations Research

mansans

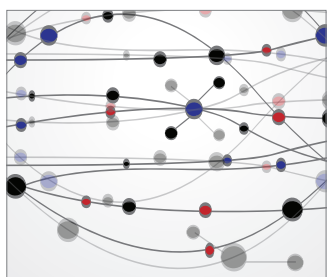

The Scientific World Journal
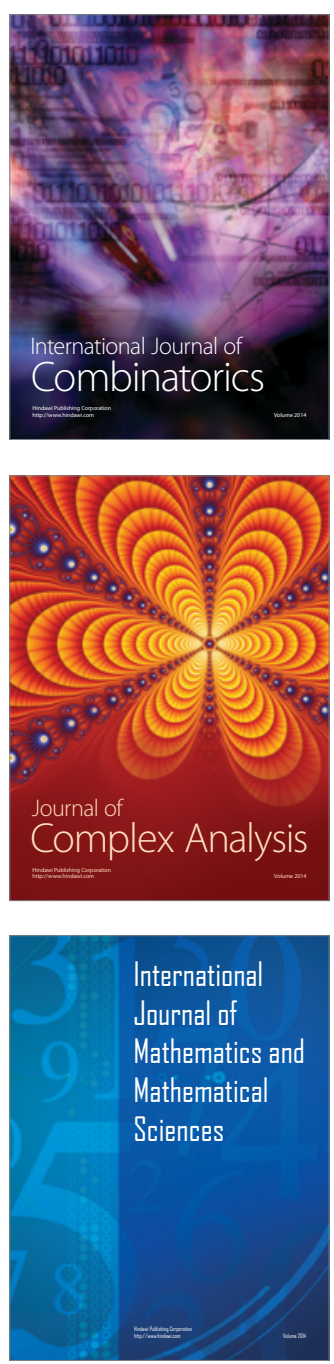
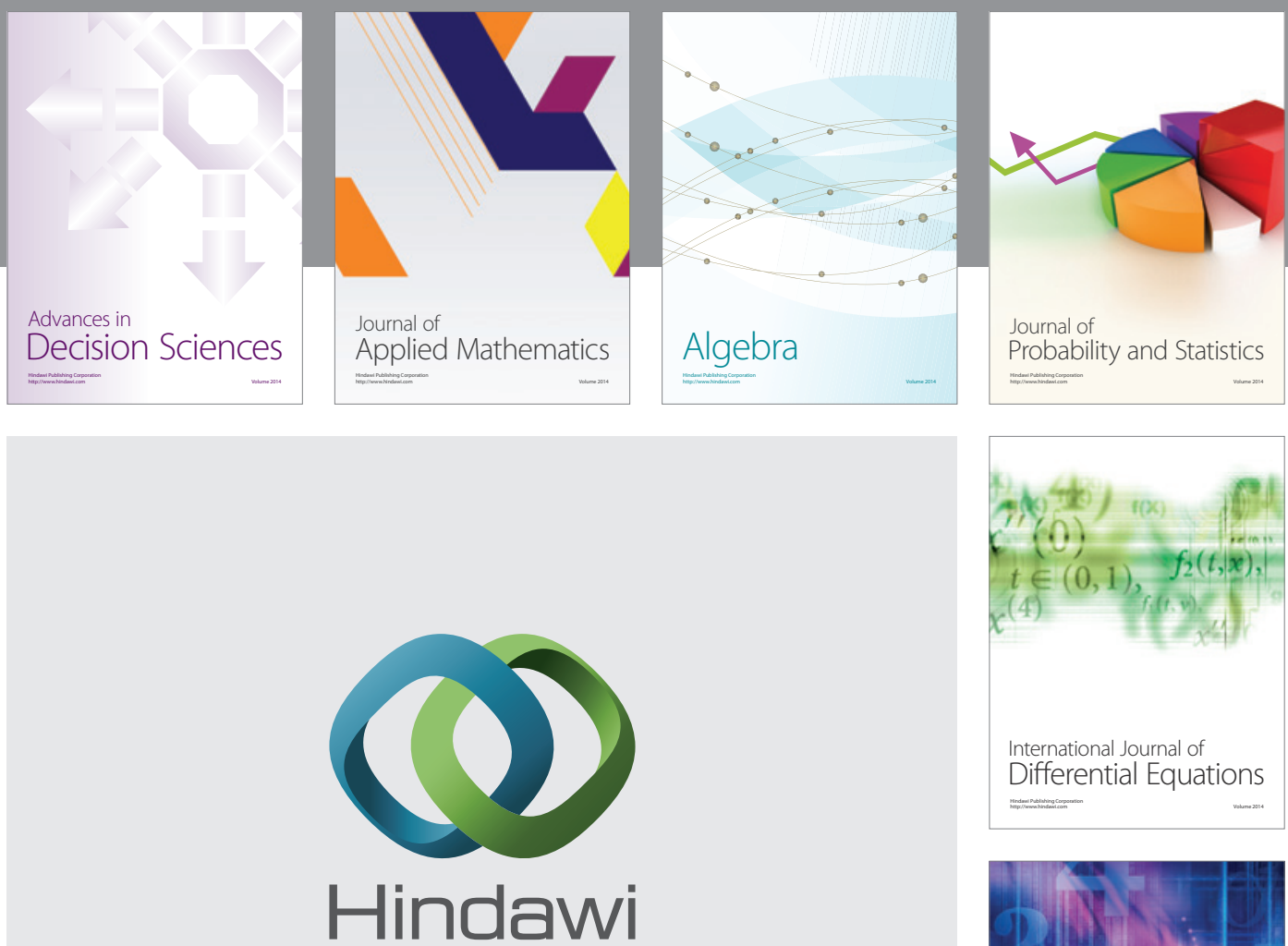

Submit your manuscripts at http://www.hindawi.com
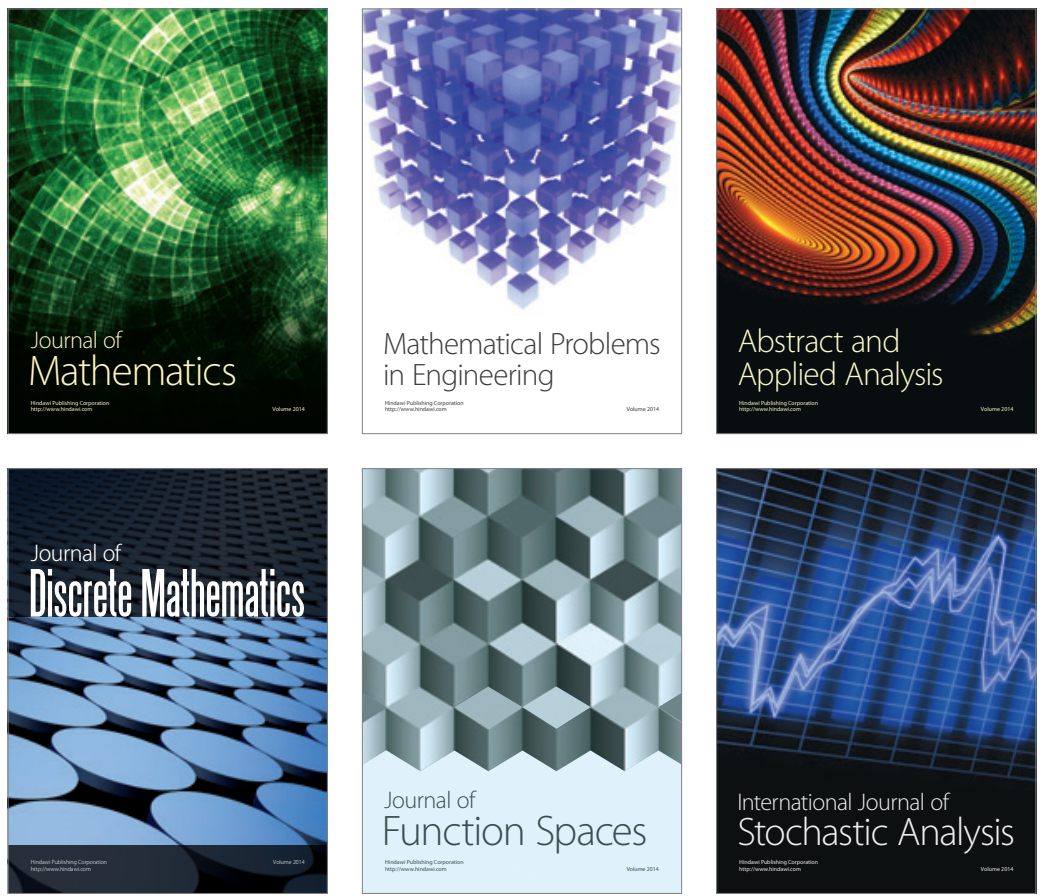

Journal of

Function Spaces

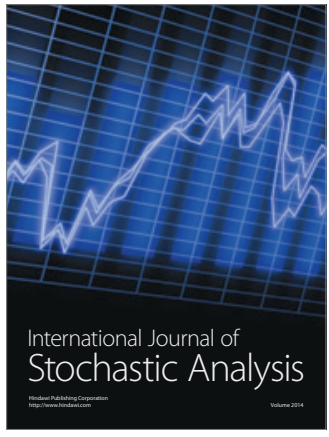

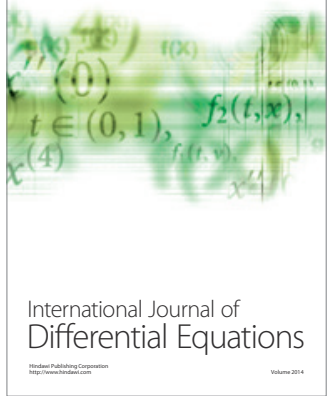
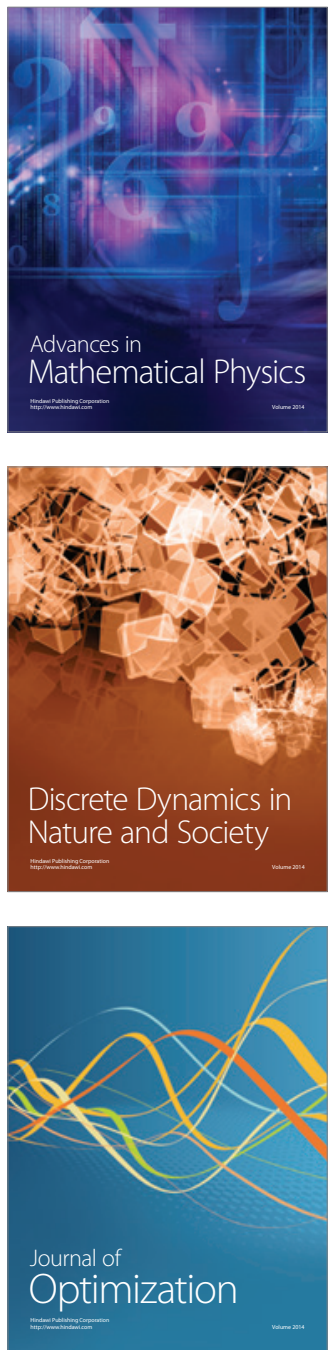